\title{
MPZL1 is highly expressed in advanced gallbladder carcinoma and promotes the aggressive behavior of human gallbladder carcinoma GBC-SD cells
}

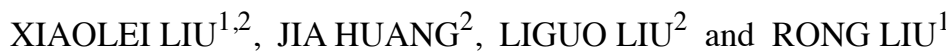 \\ ${ }^{1}$ Department of Hepato-Pancreato-Biliary Surgical Oncology, Chinese PLA General Hospital, Beijing 100853; \\ ${ }^{2}$ Department of General Surgery, China-Japan Friendship Hospital, Beijing 100029, P.R. China
}

Received July 13, 2018; Accepted May 9, 2019

DOI: $10.3892 / \mathrm{mmr} .2019 .10506$

\begin{abstract}
Myelin protein 0-like 1 (MPZL1) has been reported to have a role in hepatocellular carcinoma. However, to the best of our knowledge, there have been no studies on the function and molecular mechanism of MPZL1 gene in gallbladder carcinoma. The present study confirmed that MPZL1 was upregulated in four gallbladder carcinoma tissues according to the mRNA microarray analysis. The results of the immunohistochemical analysis of tissues from 82 patients with gallbladder carcinoma demonstrated that patients with advanced tumor stages (both $\mathrm{T}$ and $\mathrm{N}$ stage) had higher positive expression of MPZL1. Moreover, a total of 20 cases of gallbladder carcinoma and matched paired paracarcinoma tissues along with 20 samples of healthy gallbladder tissue from patients with cholecystitis were analyzed using reverse transcription-quantitative PCR and western blotting. The results demonstrated that the expression of MPZL1 in gallbladder carcinoma tissues was significantly higher than that of paired paracarcinoma tissues and randomly matched normal gallbladder epithelial tissues. According to the Tumor-Node-Metastasis classification, the expression level of MPZL1 protein in stage IV gallbladder carcinoma was significantly higher than that in stage III gallbladder carcinoma. The enhanced expression of MPZL1 gene appeared to improve the migration ability of GBC-SD cells. Conversely, GBC-SD cells that transfected with MPZL1 siRNA exhibited decreased migration ability. The results of
\end{abstract}

Correspondence to: $\mathrm{Dr}$ Rong Liu, Department of HepatoPancreato-Biliary Surgical Oncology, Chinese PLA General Hospital, 28 Fuxing Road, Beijing 100853, P.R. China

E-mail: liurong301@126.com

Abbreviations: MPZL1, myelin protein 0-like 1; ITIMs, immunoreceptor tyrosine-based inhibitory motifs; SHP2, Src homology phosphatase type-2; PTP, protein tyrosine phosphatases; CCK, Cell Counting Kit; RT-qPCR, reverse transcription-quantitative PCR

Key words: MPZL1, gallbladder carcinoma, proliferation, invasion, metastasis, apoptosis proliferation experiments showed that the knockdown of MPZL1 siRNA caused impairments in GBC-SD cell proliferation. On the contrary, the overexpression of MPZL1 increased the proliferation ability of GBC-SD cells. The results of flow cytometry analyses indicated that the upregulation of MPZL1 had an anti-apoptotic effect on GBC-SD cells. In conclusion, the present study showed that the expression and protein levels of MPZL1 were significantly higher in gallbladder carcinoma tissues, especially in patients diagnosed with advanced tumor stages. Overexpression of MPZL1 may have promoted the invasion, metastasis, proliferation and survival of GBC-SD cells.

\section{Introduction}

Gallbladder carcinoma is one of the tumor types with the worst prognosis globally, with an average survival length of $\sim 6$ months and a 5 -year survival rate $<5 \%$ (1). There are two main factors associated with the poor prognosis. Firstly, gallbladder carcinoma is not sensitive to traditional chemotherapy or radiotherapy and surgery is the only effective treatment However, due to the lack of symptoms in the early stages of the disease, most patients do not have the opportunity to receive a surgical treatment (2). Secondly, the biological characteristics of gallbladder carcinoma lead to a high possibility of recurrence or metastasis after surgery (3). Recent studies have shown that various signaling pathways are associated with the development of gallbladder carcinoma, including Hedgehog, PI3K/AKT/mTOR, Notch, Erb-b receptor tyrosine kinases (ErbB) and the mitogen-activated protein kinase (MAPK)/ERK pathway (4). However, their underlying mechanisms remain unclear.

Myelin protein 0-like 1 (MPZL1), also known as PZR, is a surface glycoprotein. It belongs to the immunoglobulin superfamily and has an extracellular segment with significant sequence homology with myelin protein 0 (a major structural protein of myelin) (5-8). There are two immunoreceptor tyrosine-based inhibitory motifs (ITIMs) in the intracellular domain of MPZL1 that specifically interact with Src homology phosphatase type-2 (SHP2) (6). SHP2 is a tyrosine phosphatase containing two Src homology 2 domains encoded by the protein tyrosine phosphatase non-receptor type 11 
(PTPN11) gene (9). Studies have confirmed that SHP2 is a cancer-promoting protein $(10,11)$. Mutations in the PTPN11 gene result in a variety of hematological malignancies, juvenile myelomonocytic leukemia, and in the development of certain solid tumors (12). SHP2 is thought to be regulatory protein affecting tumor cell proliferation, differentiation and survival through to a variety of signaling pathways, including Ras/Raf/MEK/ERK, Janus kinase (JAK)-STAT, $\mathrm{PI} 3 \mathrm{~K} / \mathrm{AKT} / \mathrm{mTOR}$ and Wnt/ $\beta$-catenin signaling pathways (13).

As a highly conserved protein, MPZL1 is expressed in a variety of cells, suggesting that it may have an important role in basic cellular functions. A previous study found that MPZL1 protein may promote the fibronectin-dependent cell migration process by recruiting and activating SHP2 protein (7). The binding of intracellular ITIMs of MPZL1 protein to SHP2 may regulate cell migration, as MPZL1 lacking ITIMs does not promote cell migration (8). Recent studies have also shown that MPZL1 may promote the fibronectin-dependent migration of mouse embryonic fibroblasts $(8,14)$, and may be involved in adhesion-dependent signaling $(15,16)$. However, the functional roles and clinical implications of MPZL1 amplification and overexpression in human cancers are largely unknown.

There are reports regarding role of MPZL1 gene in hepatocellular carcinoma. Jia et al (17) revealed that MPZL1 gene was upregulated in liver cancer tissue, and its expression was associated with clinical pathological parameters, such as age, histological grade and intrahepatic metastasis of patients with liver cancer. It also revealed that MPZL1 can activate cortactin and promote the migration and metastasis of hepatoma cells via the Src-mediated signaling pathway (17). Previous studies have also reported that MPZL1 mediates the phosphorylation of cortactin by increasing the phosphorylation levels of Src kinase, which may in turn lead to the development of distant metastases of hepatocellular carcinoma, such as lung metastasis $(5,17,18)$. Therefore, it may be possible that the upregulation of MPZL1 may affect the invasiveness or metastatic ability of gallbladder carcinoma cells.

To the best of our knowledge, there have been no studies on the function and molecular mechanism of MPZL1 in gallbladder carcinoma. Therefore, the present study aimed to elucidate the function of MPZL1 expression in gallbladder carcinoma tissues, and to further understand the effect of MPZL1 expression on gallbladder carcinoma cell functions, including proliferation, invasion, metastasis and apoptosis.

\section{Materials and methods}

Patients and tissue specimens. From January 2011 to June 2017, 82 patients (male:female ratio, 52:30; median age, 63 years; age range, 44-77 years) with gallbladder carcinoma that received surgical treatment at the Department of General Surgery, China-Japan Friendship Hospital (Beijing, China) enrolled in the present study. The carcinoma and paracarcinoma samples collected from all 82 patients were stored in paraffin in the Department of Pathology. From January 2016 to June 2017, an additional 20 paired samples of gallbladder carcinoma and adjacent healthy tissue from 20 (male:female, 13:7; median age, 63 years; age range, 44-75 years) of the 82 patients were snap frozen at $-80^{\circ} \mathrm{C}$. Moreover, from January 2017 to June 2017 , gallbladder samples of 20 patients (male:female ratio, 12:8; median age, 62 years; age range, $45-73$ years) with benign cholecystitis who received surgical treatment were also collected and frozen as a normal control group. The pathological diagnosis of all the 82 patients with gallbladder carcinoma and the 20 patients with benign cholecystitis was clear. None of the patients with gallbladder carcinoma that participated in the present study had received radiotherapy, chemotherapy or any other treatment prior to surgery. The Clinical Research Ethics Committee of Chinese PLA General Hospital and China-Japan Friendship Hospital approved the present study. All patients provided written informed consent for the use of their medical records and tissue specimens for research purposes.

$R N A$ isolation and reverse-transcription-quantitative $P C R$ $(R T-q P C R)$. Total RNA was isolated from tissue and GBC-SD cells by using the Qiazol reagent (Qiagen $\mathrm{GmbH}$ ). The concentration of total RNA was determined via A260 measurement using the ND-1000 NanoDrop spectrophotometer (NanoDrop; Thermo Fisher Scientific, Inc). A total of $500 \mathrm{ng}$ total RNA was reverse transcribed using the High Capacity cDNA Reverse Transcription kit (Thermo Fisher Scientific, Inc.). The RT temperature protocol was $25^{\circ} \mathrm{C}$ for $10 \mathrm{~min}, 37^{\circ} \mathrm{C}$ for $120 \mathrm{~min}$ and $85^{\circ} \mathrm{C}$ for $5 \mathrm{~min}$, followed by maintenance at $4^{\circ} \mathrm{C}$. A total of $10 \mathrm{ng}$ cDNA were used for qPCR using Power SYBR Green PCR Master Mix (Applied Biosystems; Thermo Fisher Scientific, Inc.), as per the manufacturer's instructions. The thermocycling conditions were as follows: Initial denaturation at $95^{\circ} \mathrm{C}$ for $10 \mathrm{~min}$; followed by 55 cycles of denaturation at $95^{\circ} \mathrm{C}$ for $30 \mathrm{sec}$ and $60^{\circ} \mathrm{C}$ for $1 \mathrm{~min}$; finally, annealing and elongation was performed by increasing the temperature from $55^{\circ} \mathrm{C}$ to $95^{\circ} \mathrm{C}$ (30 sec/degree increase). The primer sequences were as follows: MPZL1, forward 5'-TGGGCTGGAGACCTT GAC-3' and reverse 5'-CCCACCACTACCCAAACTG-3'; $\beta$-actin, forward 5'-CTTAGTTGCGTTACACCCTTTCTT G-3' and reverse 5'-CTGTCACCTTCACCGTTCCAGTTT-3'. The experiment was repeated three times and transcript levels were normalised to $\beta$-actin levels. Transcript expression levels were calculated using the $2^{-\Delta \Delta \mathrm{Cq}}$ method (19).

mRNA microarray analysis. Total RNA (100 ng) from frozen paired carcinoma and para-carcinoma tissues $(n=4$, samples selected from the 20 patients cohort) were used for the expression analysis using Prime View Human Gene Expression Array (Affymetrix; Thermo Fisher Scientific, Inc.), according to the manufacturer's instructions. Briefly, the GeneChip ${ }^{\mathrm{TM}}$ 3'IVT labeling kit (Affymetrix; Thermo Fisher Scientific, Inc.) was employed to label total RNA with biotin. To select genes for microarray analysis, the GSE62335 microarray data were downloaded from the Gene Expression Omnibus database, which was deposited by Ma et al in 2014 (20). According to the expression profile database, genes related to pathways and transcriptional misregulation in cancer were selected. The chips were hybridized overnight with, washed and stained using the GeneChip $^{\mathrm{TM}}$ Hybridization, Wash and Stain kit (Affymetrix; Thermo Fisher Scientific, Inc.). The chip was scanned using the GeneChip ${ }^{\mathrm{TM}}$ Scanner 3000 (Affymetrix; Thermo Fisher Scientific, Inc.). Gene Ontology (GO) and Kyoto Encyclopedia of Genes and Genomes (KEGG) pathway analyses were used to detect differentially expressed genes. Genes were selected for further evaluation if fold change was $>2$ and $\mathrm{P}<0.05$. 
Immunohistochemical analysis. A total of 82 paraffin fixed carcinoma tissues from gallbladder carcinoma patients were used for the immunohistochemical analysis. The 20 paired frozen samples of gallbladder carcinoma and 20 frozen gallbladder tissues in normal control group were also used for immunohistochemical analysis. The tissue sections were deparaffinized in xylene twice for $5 \mathrm{~min}$ at room temperature, and rehydrated using a graded ethanol series $(100,95$, 80 and $70 \% ; 5 \mathrm{~min}$ at room temperature for each concentration). Subsequently, the endogenous peroxidase activity was blocked by soaking in $0.3 \%$ hydrogen peroxide for $10 \mathrm{~min}$ at room temperature. Thereafter, the sections were processed in $10 \mathrm{mmol} / 1$ citrate buffer ( $\mathrm{pH} 6.0$ ) and were heated to $121^{\circ} \mathrm{C}$ in an autoclave for $20 \mathrm{~min}$ for antigen retrieval. After rinsing with PBS (pH 7.2), 10\% goat serum (Invitrogen; Thermo Fisher Scientific, Inc.) was added and incubated at room temperature for $1 \mathrm{~h}$ to block non-specific reactions. The sections were then incubated overnight at $4^{\circ} \mathrm{C}$ with MPZL1 monoclonal antibody (cat. no. ab151541; diluted 1:100; Abcam). Subsequently, $25 \mu \mathrm{l}$ horseradish peroxidase (HRP)-labeled secondary antibody (goat anti-rabbit IgG H\&L HRP; cat. no. ab205718; diluted 1:100; Abcam) was added to each section for $30 \mathrm{~min}$ at room temperature. Each section was then soaked in PBS for $5 \mathrm{~min}$ and rinsed three times. All slides were processed using the peroxidase anti-peroxidase method. After rinsing with PBS, the peroxidase reaction was visualized by incubating the sections with 3,3-diamino-benzidine tetrahydrochloride for $5 \mathrm{~min}$ at room temperature. The sections were then rinsed with water, counterstained with hematoxylin (5\%) for $1 \mathrm{~min}$ at room temperature, dehydrated and coverslipped. All of the immunostained sections were evaluated in a blinded manner by two independent and experienced observers without any knowledge on the clinicopathological features of the patients. Five fields (magnification, x400) of each specimen were selected randomly, and nuclear staining was examined using a light microscope. For the interpretation of MPZL1 staining results, the nuclear MPZL1 expression was scored as a percentage of positive cells by two pathologists. Carcinoma tissues, paracarcinoma tissues and gallbladder tissues in the normal control group showing nuclear MPZL1 expression in $\geq 5 \%$ of cells were considered to be MPZL1-positive.

Cell culture. The human gallbladder carcinoma GBC-SD cell line was purchased from the Type Culture Collection of the Chinese Academy of Sciences (Shanghai, China). Cells were maintained in RPMI-1640 medium supplemented with $10 \%$ heat-inactivated fetal calf serum, $2 \mathrm{mM} \mathrm{L-glutamine,} \mathrm{and} 100 \mathrm{U} / \mathrm{ml}$ penicillin and streptomycin mixture (all from Gibco; Thermo Fisher Scientific, Inc.), and incubated at $37^{\circ} \mathrm{C}$ with $5 \% \mathrm{CO}_{2}$.

Western blot analysis. Tissue specimens and GBC-SD cell were lysed in the lysis buffer $(1 \mathrm{M}$ Tris- $\mathrm{HCl}$ at $\mathrm{pH} 7.5,1 \%$ Triton X-100, $1 \%$ Nonidet P-40, 10\% SDS, $0.5 \%$ sodium deoxycholate, $0.5 \mathrm{M}$ EDTA, $10 \mu \mathrm{g} / \mathrm{ml}$ leupeptin, $10 \mu \mathrm{g} / \mathrm{ml}$ aprotinin and $1 \mathrm{mM}$ phenylmethylsulfonyl fluoride) and then centrifuged at $4^{\circ} \mathrm{C}$ and $10,000 \times \mathrm{g}$ for $30 \mathrm{~min}$ to collect the supernatant. Protein concentration was determined using the Bio-Rad protein assay kit (Bio-Rad Laboratories, Inc.). Equal amounts $(60 \mu \mathrm{g} /$ lane $)$ of protein were separated by SDS-PAGE on $10 \%$ gels, and transferred to polyvinylidine difluoride
(PVDF) membranes (EMD Millipore). Membranes were then blocked with $5 \%$ non-fat milk in TBS with $0.05 \%$ Tween-20 for $2 \mathrm{~h}$ at room temperature. Subsequently, the membrane was incubated for $12 \mathrm{~h}$ at $4^{\circ} \mathrm{C}$ with primary antibodies, MPZL1 monoclonal antibody (cat. no. ab151541; diluted 1:100; Abcam) for $1 \mathrm{~h}$ at room temperature. In addition, $\beta$-actin antibody (cat. no. WL01845; WanleiBio) diluted in 5\% non-fat milk $(1: 1,000)$ was used to incubate the PVDF membrane at $4^{\circ} \mathrm{C}$ overnight. Subsequently, the PVDF membrane was washed in TBS under agitation for $5 \mathrm{~min}$; this step was repeated four times. The membrane was then incubated with a secondary antibody (goat anti-rabbit IgG HRP; cat. no. WLA023; diluted 1:5,000; WanleiBio) diluted in 5\% non-fat milk at $37^{\circ} \mathrm{C}$ for $45 \mathrm{~min}$. Immunoblotting bands were visualized via enhanced chemiluminescence (NEN Life Science Products; PerkinElmer, Inc.). The band density was measured using a computer-assisted image analysis system (Image Lab Software version 5.2.x; Bio-Rad Laboratories, Inc.), and band densities were normalized against the $\beta$-actin levels.

MPZL1 overexpression and silence. TRIzol ${ }^{\circledR}$ was used to extract total RNA from normal gallbladder epithelium. Total RNA was the reverse transcribed using the High Capacity cDNA Reverse Transcription kit (Applied Biosystems; Thermo Fisher Scientific, Inc.) to obtain human tissue cDNA library. The primer sequences used to amplify the MPZL1 gene containing the sites for HindIII and XbaI were: Forward 5'-AAGCTTCAGGTGGCGGAGAGATCAGAAG-3' and reverse 5'-TCTAGAGACTTGTCCTTGCCTGGGTCTC-3'. PCR was then performed to amplify the cDNA sequence with restriction sites using Platinum Taq DNA Polymerase (cat. no. 10966034, Invitrogen; Thermo Fisher Scientific, Inc.); the thermocycling conditions were the same as for RT-qPCR., Subsequently $1 \%$ agarose gel electrophoresis was performed to recover and purify the amplified cDNA fragment of interest, and DNA was visualized using a gel imaging system (E-Gel Imager; Invitrogen; Thermo Fisher Scientific, Inc.). After enzymatic digestion and purification of the PCR amplification product, it was cloned into a pCMV plasmid vector (cat. no. V51020; Invitrogen; Thermo Fisher Scientific, Inc.) using a T4 DNA ligase to connect the target sequence to the pCMV vector, thus generating the pCMV-MPZL1 high expression vector. After transforming DH5 $\alpha$ competent cells (cat. no. 18263012 Invitrogen; Thermo Fisher Scientific, Inc.) with the pCMV-MPZL1, a single clone was selected and the correct construction of the vector was ensured by sequencing.

Three pairs of small interfering RNA (siRNA) probes targeting the MPZL1 mRNA were designed (WanleiBio) using the chemical synthesis method (21), and their transfection efficiency was verified. One pair of siRNA molecules that was the most efficient at silencing the MPZL1 mRNA (siRNA-1: 5'-GCACCUAUAUCUGUGAUGUTT-3'; siRNA-2: 5'-GUCAGAGUCUGUGGUGUAUTT-3'; siRNA-3: 5'-GAG AGUUUGUCACCAGUUATT-3') was selected. The pCMV-MPZL1 vector, blank vector, siMPZL1 and scramble RNA (5'-CCCACCACTACCCAAACTG-3') were transfected into GBC-SD cells using Lipofectamine 2000. The concentration of transfected DNA was $0.02 \mu \mathrm{g} / \mu \mathrm{l}$ and a total of $100 \mu \mathrm{l}$ was added to GBC-SD cells. Subsequent experiments were performed $16 \mathrm{~h}$ post-transfection. 
Cell proliferation assay. Cell proliferation was measured using Cell Counting Kit-8 (CCK-8; Dojindo Molecular Technologies, Inc.), following the manufacturer's instructions. Briefly, GBC-SD cells were seeded onto 96-well cell culture plates at a concentration of $2 \times 10^{4}$ cells/well in $100 \mu \mathrm{l}$ fresh complete medium (cat. no. 12558011; AmnioMax ${ }^{\mathrm{TM}} \mathrm{C} 100$; Gibco). CCK-8 reagent $(10 \mu \mathrm{l})$ was added to a subset of wells and incubated for $2 \mathrm{~h}$ at $37^{\circ} \mathrm{C}$, and the absorbance was measured using an automated plate reader under the detection wavelength of $450 \mathrm{~nm}$. Each measurement was performed in triplicate and the experiments were repeated twice.

Cell migration and invasion assay. Matrigel gel was thawed overnight at $4^{\circ} \mathrm{C}$. The Matrigel gel was placed on ice in a clean bench and diluted 1:3 in serum-free medium. The Transwell chamber were placed in 24 -well plates, $40 \mu 1$ pre-diluted Matrigel were added to the chamber membrane and placed in a $37^{\circ} \mathrm{C}$ incubator for $2 \mathrm{~h}$. The transfected GBC-SD cells were cultured in 6-well plates $\left(2 \times 10^{4}\right.$ cells/well $)$ at $37^{\circ} \mathrm{C}$ in an atmosphere containing $5 \% \mathrm{CO}_{2}$ and saturated humidity for $48 \mathrm{~h}$. The culture medium from each group of cells was discarded, cells were washed three times with PBS before being detached with $0.25 \%$ trypsin. Serum-free medium was then added to create a single cell suspension. A total of $800 \mu$ l culture medium containing $30 \%$ FBS was added to lower chamber of the 24-well Matrigel-coated Transwell, and $200 \mu 1$ cell suspension $\left(2 \times 10^{4}\right.$ cells/well $)$ were added to the upper chamber. The 24-well plate was incubated in a cell incubator at $37^{\circ} \mathrm{C}$ under $5 \% \mathrm{CO}_{2}$ and saturated humidity for $12 \mathrm{~h}$. Subsequently, the lower chamber was washed twice with PBS, fixed with $4 \%$ paraformaldehyde at room temperature for $20 \mathrm{~min}$, stained with $0.5 \%$ crystal violet dye at room temperature for $5 \mathrm{~min}$, and rinsed with distilled water. The cells that migrated to the lower layer of the micro-porous membrane were counted under an inverted light microscope (magnification, x200). The number of cells in each sample was the average number of cells across five fields.

Cell apoptosis assay. GBC-SD cell apoptosis was evaluated using an Annexin V-FITC/propidium iodide (PI) staining assay. Flow cytometry analysis of apoptotic cells was performed using an Annexin V-FITC/PI staining kit (BD Biosciences). After washing with cold PBS, the cells were re-suspended in binding buffer (100 mM HEPES, pH 7.4, $100 \mathrm{mM} \mathrm{NaCl}$ and $25 \mathrm{mM} \mathrm{CaCl}_{2}$ ) followed by staining with Annexin V-FITC/PI at room temperature in the dark for $15 \mathrm{~min}$. Apoptotic cells were then evaluated by gating PI and Annexin V positive cells on a FACSCalibur (BD Biosciences); data were analyzed using CellQuest Pro software, version 5.1 (BD Biosciences). Annexin $\mathrm{V}$ was set in the horizontal axis and PI was set in the vertical axis. Early apoptotic cells were located in the lower right quadrant of the flow cytometry plot.

Statistical analysis. Statistical analyses were performed using SPSS software (version 20.0, IBM Corp.). Categorical variables were compared using a $\chi^{2}$ or Fisher's exact test. A Student's $t$ test was applied for continuous variables with a normal distribution. Multiple groups were compared using the Bonferroni-corrected one-way ANOVA test. For paired samples, a paired Student's t-test was applied.
Table I. Results of immunohistochemical analysis of samples from patients with gallbladder carcinoma.

\begin{tabular}{|c|c|c|c|c|}
\hline \multicolumn{5}{|c|}{ A, $T$ stage $(n=82)$} \\
\hline \multirow[b]{2}{*}{ Group } & \multicolumn{3}{|c|}{ Number of patients } & \multirow[b]{2}{*}{ P-value } \\
\hline & All & MPZL1 (-) & MPZL1 (+) & \\
\hline II & 13 & 9 & 4 & 0.010 \\
\hline III & 34 & 15 & 19 & \\
\hline IV & 35 & 8 & 27 & \\
\hline
\end{tabular}

B, N stage $(n=82)$

\begin{tabular}{lcccc}
\hline & \multicolumn{3}{c}{ Number of patients } & \\
\cline { 2 - 4 } Group & All & MPZL1 (-) & MPZL1 (+) & P-value \\
\hline Negative & 31 & 21 & 10 & $<0.001$ \\
Positive & 51 & 11 & 40 & \\
\hline
\end{tabular}

MPZL1, myelin protein 0-like 1.

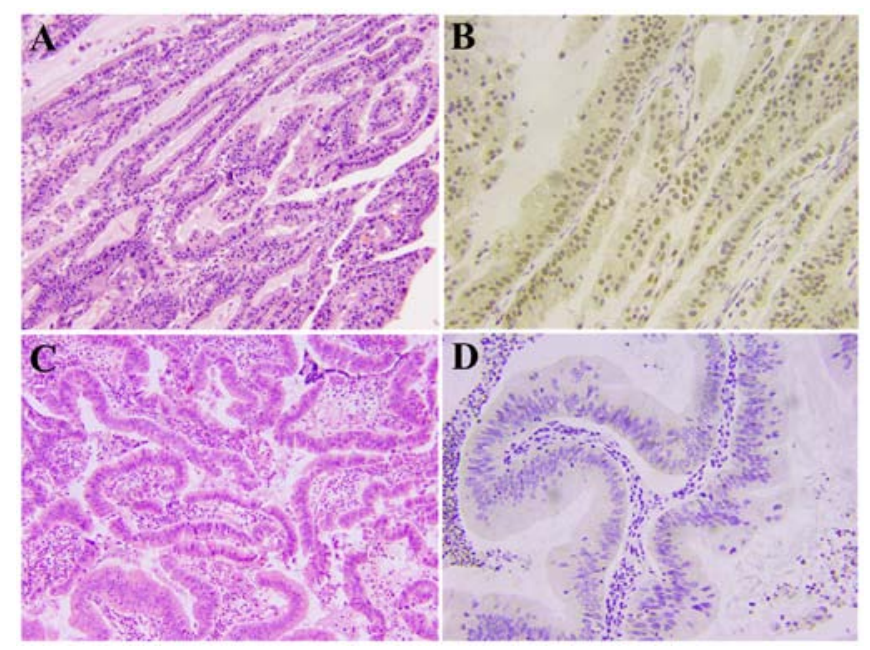

Figure 1. Histopathological images of gallbladder carcinoma. Histological images of gallbladder samples from patients diagnosed with T4 stage gallbladder carcinoma (A) stained with hematoxylin and eosin and (B) immunohistochemistry to detect MPZL1. Gallbladder samples from patients diagnosed with T2 stage gallbladder carcinoma, (C) stained with hematoxylin and eosin and (D) immunohistochemistry against MPZL1. Generally, T4 stage samples show more MPZL1-positive cells than T2 samples (A and B: magnification x200, C and D: magnification x400). MPZL1, myelin protein 0-like 1 .

Bonferroni-corrected Student's t-test was used to compare the paired carcinoma and paracarcinoma tissues with control tissues. $\mathrm{P}<0.05$ was considered to indicate a statistically significant difference.

\section{Results}

Results of mRNA microarray analysis of gallbladder carcinoma tissues and immunohistochemical analysis in different 


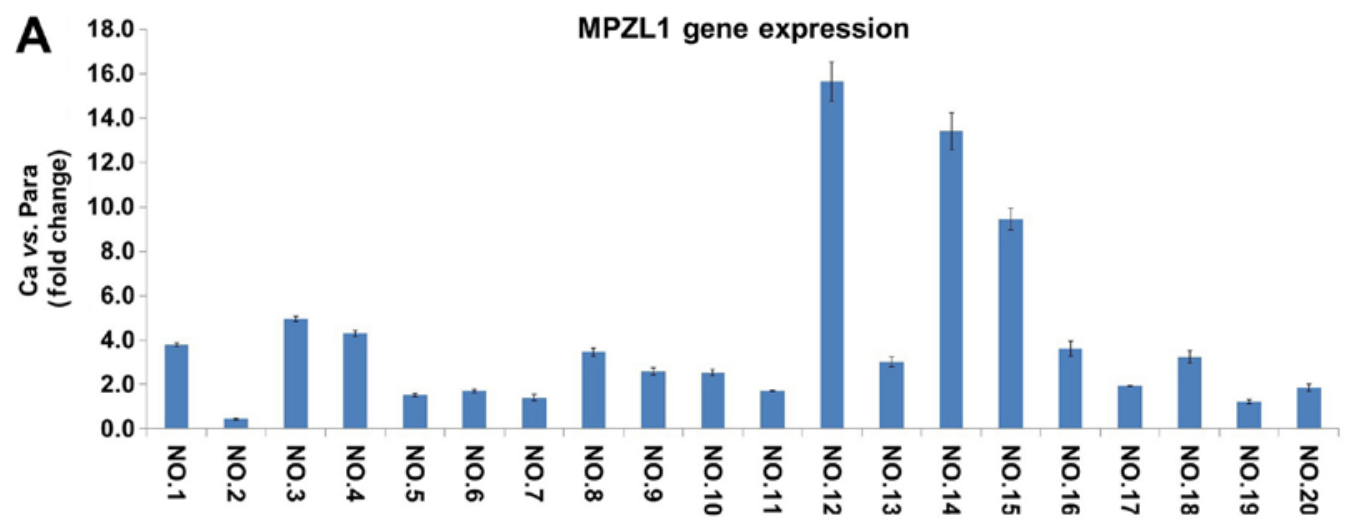

B
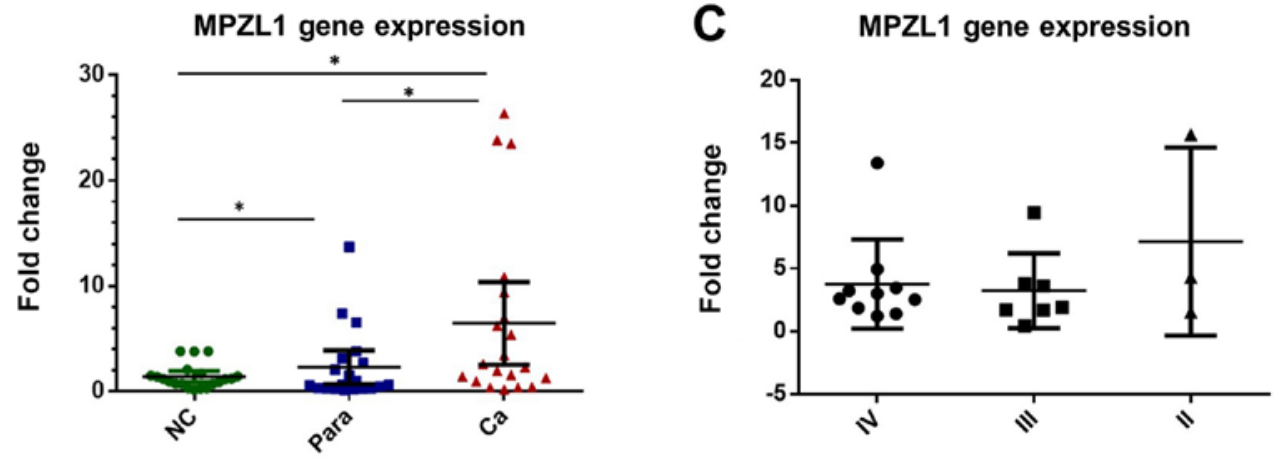

Figure 2. Reverse transcription-quantitative PCR analysis of MPZL1 expression in normal, carcinoma and matched paraneoplastic gallbladder tissues. (A) Expression of the MPZL1 gene RNA level in each of the 20 gallbladder carcinoma tissues samples relative to matched paracarcinoma tissues. Overall, MPZL1 is higher in the neoplastic tissue. (B) Comparison of MPZL1 expression between neoplastic, paraneoplastic and healthy gallbladder tissue. Compared with the paracarcinoma and normal gallbladder samples, MPZL1 was highly expressed in gallbladder carcinoma samples. (C) Expression of MPZL1 in gallbladder carcinoma tissues at different TNM stages. To calculate the fold change, $\beta$-actin was used as the control. "P<0.05. MPZL1, myelin protein 0-like 1; NC, normal gallbladder epithelium (control); Para, matched paracarcinoma tissue; Ca, gallbladder carcinoma.

Tumor-Node-Metastasis (TNM) stages. Of the 20,000 genes that were detected, genes were selected for further evaluation if the fold change was $>2$, and $\mathrm{P}<0.05$. Using these criteria, 580 upregulated and 999 downregulated genes were identified in gallbladder carcinoma tissues. Through GO analysis and KEGG pathway analysis of differentially expressed genes, a total of 18 genes involved in tyrosine phosphorylation were identified, of which 2 were downregulated and 16 were upregulated, including MPZL1.

A total of 82 paraffin fixed tissues from gallbladder carcinoma patients were collected for the immunohistochemical analysis. The final pathological results of TNM staging were also evaluated. The positive rates of MPZL1 across the different $\mathrm{T}$ stages were $30.8 \%$ (4/13, T2), 55.9\% (19/34, T3) and $77.1 \%(27 / 35, \mathrm{~T} 4)$, respectively and the difference between these groups was significant $(\mathrm{P}<0.01)$. The positive rates of MPZL1 across the different $\mathrm{N}$ stages were $32.3 \%$ (10/31, $\mathrm{N}$ negative) and $78.4 \%$ (40/51, $\mathrm{N}$ positive) and the difference between them was also significant $(\mathrm{P}<0.01$; Table I; Fig. 1). The immunohistochemical analysis results of 20 paracarcinoma tissues and 20 normal gallbladder epithelial tissues were all negative for MPZL1 expression.

MPZL1 mRNA and protein are highly expressed in gallbladder carcinoma tissues. The 20 cases of gallbladder carcinoma and paired paracarcinoma tissues were evaluated, and gallbladder tissue specimens from patients with cholecystitis were also included as normal controls. The results of RT-qPCR suggested that the expression of MPZL1 gene in gallbladder carcinoma tissues was significantly higher than that in paracarcinoma tissues (Fig. 2A). Meanwhile, the expression of MPZL1 gene in normal gallbladder epithelial tissue was significantly lower than that in gallbladder carcinoma tissues and paracarcinoma tissues $(\mathrm{P}<0.05$; Fig. 2B). There was no significant difference in the expression level of the MPZL1 gene in gallbladder carcinoma tissues with different TNM staging (Fig. 2C).

The results of the western blotting showed that the expression of MPZL1 protein in gallbladder carcinoma tissues was significantly higher than that of paired paracarcinoma tissues and randomly matched normal gallbladder epithelial tissues. Densitometry analysis of the western blot results showed that the expression of MPZL1 protein in gallbladder carcinoma was higher compared with matched paired adjacent tissues $(\mathrm{P}<0.05)$ and control gallbladder epithelial tissues $(\mathrm{P}<0.05)$, while the levels of MPZL1 protein in the paracarcinoma tissues were not markedly different from that of control tissues $(\mathrm{P}=0.23)$ (Fig. 3A). According to the different TNM staging classification, the protein levels of MPZL1 protein in stage IV gallbladder carcinoma were higher than that in stage III gallbladder carcinoma ( $\mathrm{P}<0.05 ;$ Fig. $3 \mathrm{~B})$. According to the $\mathrm{T}$ stage of gallbladder carcinoma, it was also observed that the levels of MPZL1 protein in the T4 stage were also higher than those in the $\mathrm{T} 3$ stage $(\mathrm{P}<0.05$; Fig. $3 \mathrm{C})$.

MPZL1 overexpression facilitates the invasion and metastasis of $G B C$-SD cells. An overexpression vector and MPZL1 siRNA 
A

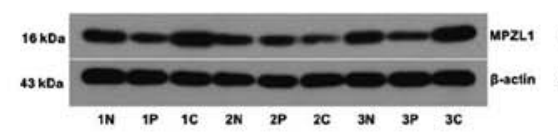

MPZL1 protein expression profiles
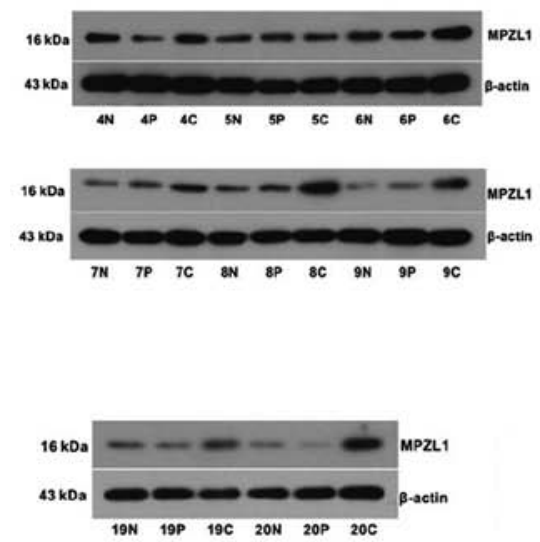
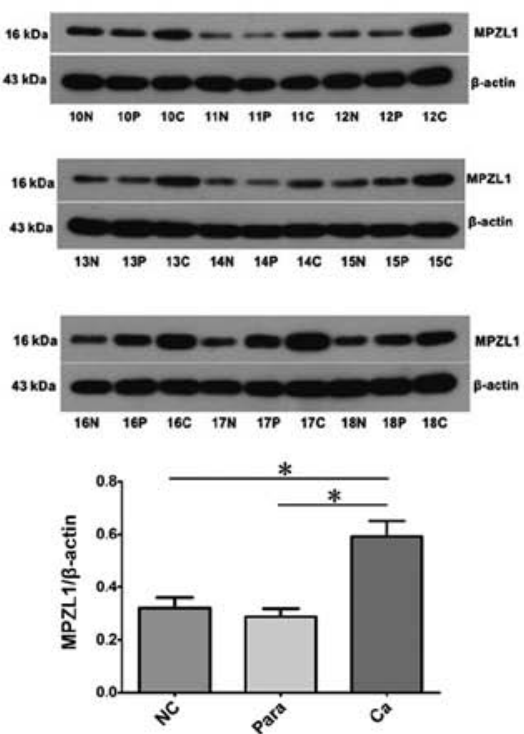

B

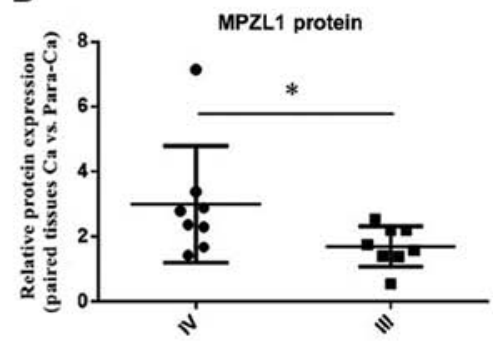

C

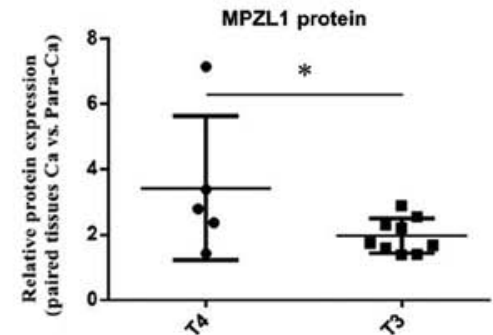

Figure 3. Protein levels of MPZL1 in normal, carcinoma and matched paraneoplastic gallbladder tissues. (A) Western blot analysis of MPZL1 protein in carcinoma, paired paracarcinoma and normal gallbladder tissue samples, using $\beta$-actin as an internal reference. (B) Densitometry analysis of MPZL1 protein levels in gallbladder carcinoma tissue specimens of stage III and stage IV according to Tumor-Node-Metastasis classification. (C) Densitometry analysis of MPZL1 protein levels in gallbladder carcinoma tissue samples of stages T4 and T3. "P<0.05. MPZL1, myelin protein 0-like 1; N, normal gallbladder epithelium (control); P, matched paracarcinoma gallbladder sample; C, gallbladder carcinoma sample; $\mathrm{Ca}$, carcinoma; Para-Ca, paracarcinoma; $\mathrm{NC}$, normal control group.

A

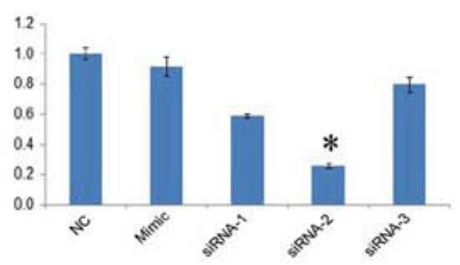

C

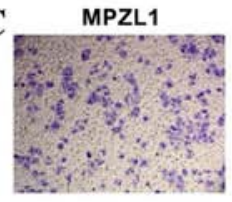

$\mathbf{E}$

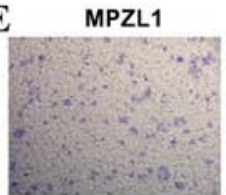

SIRNA-MPZL1

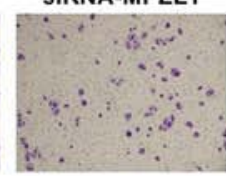

SIRNA-MPZL1

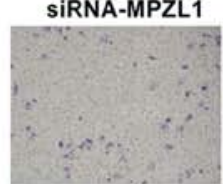

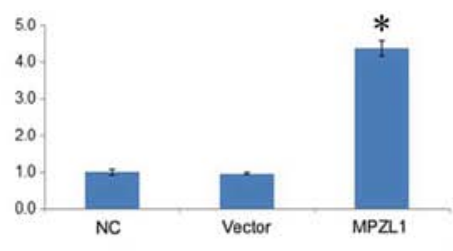

SIRNA-NC
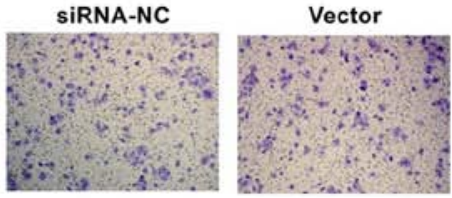

SIRNA-NC
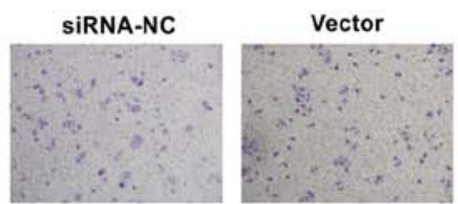

$\mathrm{B}_{16 \mathrm{kO} 2}$

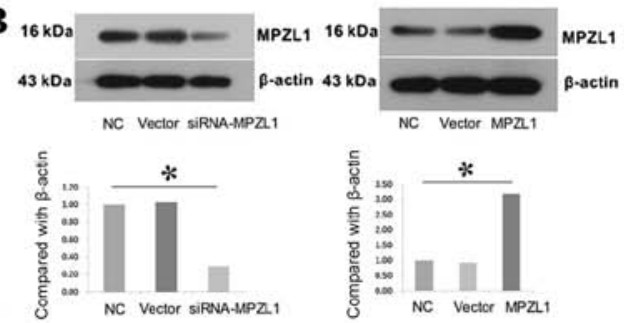

NC

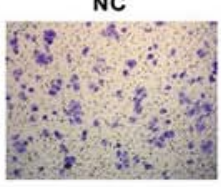

NC

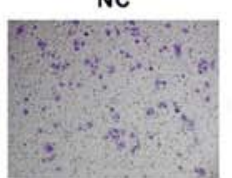

D

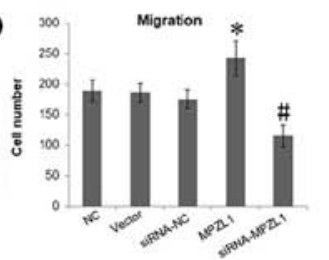

F

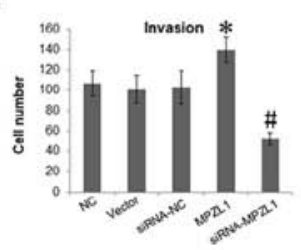

Figure 4. MPZL1 manipulation affects GBC-SD cell migration and invasion. (A) Reverse transcription-quantitative PCR and (B) western blot analysis were used to verify the expression changes in MPZL1 mRNA and protein levels in GBC-SD cells after following transfection with a MPZL1 overexpression vector or siRNA. (C) Transwell migration assays and (D) respective quantification of GBC-SD cells following MPZL1 overexpression or siRNA-mediated knockdown. MPZL1 overexpression promoted GBC-SD cell migration. (E) Transwell invasion assays and (F) respective quantification of GBC-SD cells following MPZL1 overexpression or siRNA-mediated knockdown. The results revealed that overexpression of MPZL1 gene also promoted GBC-SD invasiveness. Magnification, $x 100 .{ }^{~} \mathrm{P}<0.05$, compared with NC. ${ }^{*} \mathrm{P}<0.05$, compared with siRNA-NC. MPZL1, myelin protein 0-like 1; siRNA, small interfering RNA.

were used to change the expression of MPZL1 gene in the gallbladder carcinoma cell line GBC-SD. RT-qPCR and western blotting methods were subsequently used to verify whether the gene expression and protein levels of MZPL1 in GBC-SD cells, respectively, were affected by the overexpression vector and
siRNA. Of the three siRNAs tested, siRNA-2 exhibited the highest efficiency and was therefore selected for subsequent experiments. The results indicated that the expression levels of MPZL1 mRNA and protein in GBC-SD cells transfected with the MPZL1 overexpression vector were increased $(\mathrm{P}<0.05)$, 

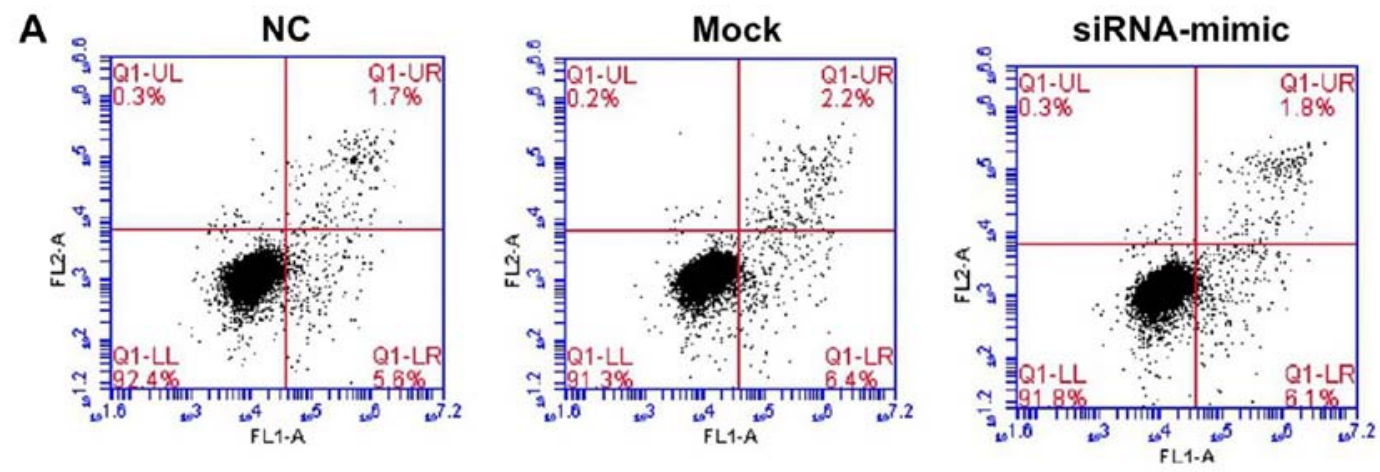

MPZL1-enhanced
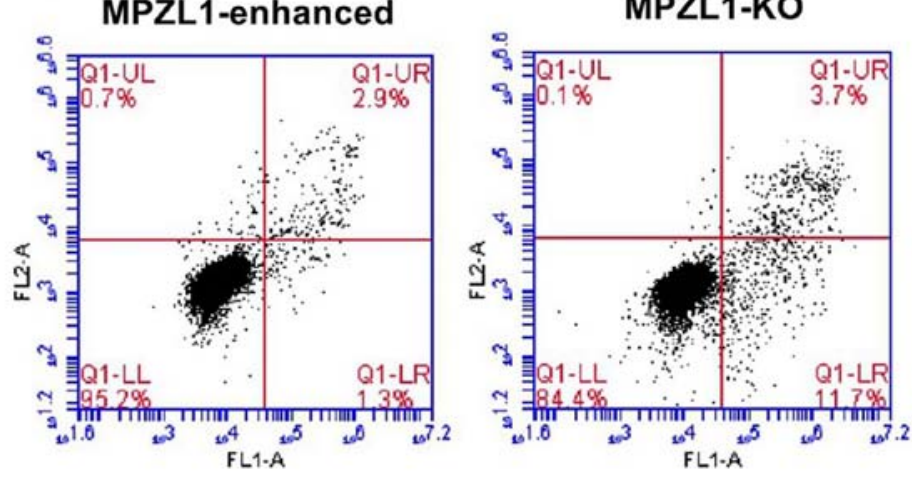

B

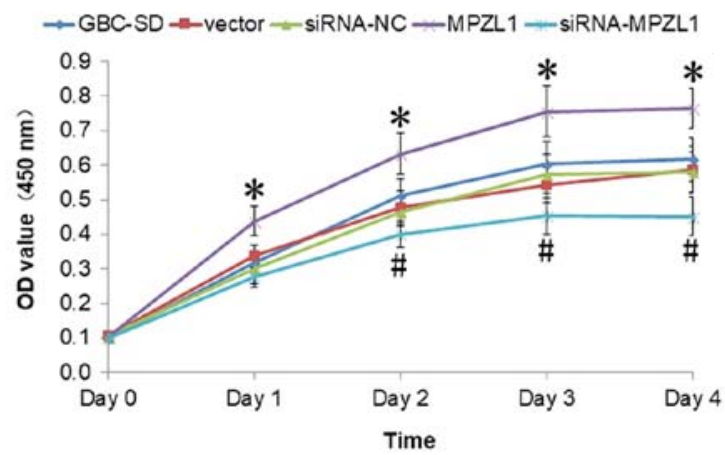

Figure 5. GBC-SD proliferation and apoptosis following MPZL1 manipulation. (A) Flow cytometry analysis of MPZL1 overexpression and knockdown on apoptosis of GBC-SD cells. Annexin V was set in the horizontal axis and PI was set in the vertical axis. (B) Effect of MPZL1 overexpression and knockdown on proliferation of GBC-SD cells. ${ }^{*} \mathrm{P}<0.05$, MPZL1 vs. GBC-SD. ${ }^{*} \mathrm{P}<0.05$, siRNA-MPZL1 vs. GBC-SD. MPZL1, myelin protein 0-like 1; siRNA, small interfering RNA.

and the levels of MPZL1 mRNA and protein in GBC-SD cells transfected with MPZL1 siRNA were decreased $(\mathrm{P}<0.05)$ (Fig. 4A and B).

Both GBC-SD cells overexpressing or underexpressing MPZL1 were evaluated in Transwell migration and invasion experiments. The results showed that enhanced expression of MPZL1 gene increased the number of migrating GBC-SD cells. Conversely, the downregulation of MPZL1 via siRNA led to a decrease in the number of migrating GBC-SD cells (Fig. 4C and D). Regarding the Transwell invasion experiments, the results confirmed that, compared with normal GBC-SD cells, overexpression of MPZL1 gene increased the invasive ability of GBC-SD cells, while MPZL1 downregulation decreased their invasive ability (Fig. 4E and F).

Enhancement of MPZL1 expression is promotes the proliferation and survival of $G B C-S D$ cells. GBC-SD cells overexpressing and downregulating MPZL1 were further tested in cell proliferation experiments. The results showed that after siRNA transfection against MPZL1, the proliferation of GBC-SD cells was significantly impaired $(\mathrm{P}<0.05)$. By contrast, MPZL1 overexpression significantly increased the proliferation ability of GBC-SD cells $(\mathrm{P}<0.05)$ (Fig. 5B). The results of flow cytometry analysis showed that only $1.3 \%$ of MPZL1 overexpressing cells were in the early apoptosis stage (Q1-LR). On the other hand, a total of $11.7 \%$ of GBC-SD cells with transfected with MPZL1 siRNA were in the early apoptosis stage. In addition, the rate of normal control cells in the early apoptosis stage was 5.6\%. The proportion of GBC-SD cells at the advanced apoptosis stage following MPZL1 overexpression was $2.9 \%$, the proportion of cells in the advanced apoptotic stage following MPLZ1 knockdown was $3.7 \%$ (Fig. 5A).

\section{Discussion}

Gallbladder carcinoma ranks as the gastrointestinal cancer with the sixth highest incidence, with an annual incidence 
rate of 2.2 per 100,000 people, and a mortality rate of 1.7 per 100,000 (1). Recent studies have also confirmed the involvement of Hedgehog, PI3K/AKT/mTOR, Notch, ErbB, MAPK/ERK and other signaling pathways in gallbladder carcinoma development and progression (4,22-25). However, the specific molecular mechanisms underlying gallbladder carcinoma remain unknown, and further research is required.

Tyrosine phosphorylation, which has an important role in the proliferation and migration of tumor cells, is a reversible and dynamic process that requires the participation of PTKs and PTPs (26). Studies have also identified mutations in PTPs followed by changes in their respective signaling pathways in a number of tumor types (27-30). Although no PTP-related drugs are yet available, studies have confirmed that PTP-associated factors, such as SHP2, dual specificity protein phosphatase 1 , acid phosphatase 1 and dual specificity phosphatases have important tumor-promoting effects, and may therefore be important anti-tumor targets (31-33). However, to the best of our knowledge, there are no reports on the role of PTP pathway-associated genes in gallbladder carcinoma. Therefore, through a microarray gene expression analysis of four gallbladder carcinoma tissue samples, the present study showed that there were eight upregulated genes in gallbladder carcinoma tissues associated with the PTP pathway, including MPZL1.

MPZL1 belongs to the immunoglobulin superfamily, containing an extracellular domain, and significant sequence homology with myelin P0 (5). There are two immunoreceptor ITIMs in the intracellular portion that specifically interact with SHP2 (6). Studies have also confirmed that SHP2 is a tumor promoting PTP (10-12), involved in the proliferation, differentiation and survival of tumor cells through a variety of signaling pathways, including Ras/Raf/MEK/ERK,JAK-STAT, $\mathrm{PI} 3 \mathrm{~K} / \mathrm{AKT} / \mathrm{mTOR}$ and $\mathrm{Wnt} / \beta$-catenin $(7,13,34,35)$. Studies have also found that MPZL1 can promote fibronectin-dependent cell migration process by recruiting and activating SHP2 (8). A study has also found that the expression of MPZL1 was upregulated in liver cancer tissues, and through cortactin activation, was able to promote the migration and metastasis of hepatoma cells through the Src-signaling pathway (22). Recently, other studies have documented a role for cortactin in the promotion of cancer cell motility and invasion, including a critical role in the development of invadopodia, actin-rich subcellular protrusions associated with the degradation of the extracellular matrix by cancer cells (36-38). Moreover, MPZL1 has also been shown to activate Src kinase upon stimulation with extracellular stimuli $(39,40)$. However, prior to the present study, the role of MPLZ1 in gallbladder carcinoma remained unknown.

The present study verified that high levels of MPZL1 in gallbladder carcinoma tissues at the gene and protein levels via $\mathrm{RT}$-qPCR and western blotting. These suggested that the expression of MPZL1 gene in gallbladder carcinoma tissues was significantly higher than that in paracarcinoma tissues and normal gallbladder epithelial tissues. The reason as to why some cases of paracarcinoma tissues showed high expression of MPZL1 mRNA may be associated with the proximity of the sampled tissue to the carcinoma area or with the biological complexity of the samples. The western blotting results also showed that MPZL1 protein levels were higher in gallbladder carcinoma tissues than paracarcinoma tissues and randomly matched normal gallbladder epithelial tissues. The levels of MPZL1 protein in T4 stage samples were seemingly higher than that the levels at the T3 stage. The results of immunohistochemical analysis also confirmed that patients with advanced gallbladder carcinoma were more likely to have higher levels of MPZL1 positive cells, which indicated that MPZL1 may be involved in the invasion and metastasis of gallbladder carcinoma cells. The result of RT-qPCR failed to detect a difference in different TNM stages, which did not match the results of western blot and immunohistochemical analyses. The reason for this may be the limited number of samples. Further experiments with a larger group may be helpful to clarify this issue. In fact, MPZL1 overexpression in GBC-SD cells confirmed that the enhanced expression of MPZL1 gene was beneficial GBC-SD cell migration and invasion. Conversely, MPZL1 knockdown in the same cell line led to a decreased migration and invasion ability. Moreover, the proliferation ability of GBC-SD cells was reduced following MPLZ1 knockdown, and the enhancement of MPZL1 gene expression promoted cell viability. Lastly, the flow cytometry analysis revealed that MPZL1 overexpression GBC-SD cells appeared to reduce the early activation of the apoptotic pathway, promoting cell survival.

In conclusion, the present findings revealed that the levels of MPZL1 gene and protein were significantly higher in gallbladder carcinoma tissues, particularly in patients with advanced tumors. Overexpression of the MPZL1 gene also promoted cancer GBC-SD cell invasion, metastasis, proliferation and antiapoptotic ability. Further studies should focus on the possible signaling pathways associated with MPZL1 to uncover the molecular mechanisms underlying the early metastasis of gallbladder carcinoma.

\section{Acknowledgements}

Not applicable.

\section{Funding}

No funding was received.

\section{Availability of data and materials}

The datasets used and/or analyzed during the current study are available from the corresponding author on reasonable request.

\section{Authors' contributions}

XL and RL conceptualized the study design and wrote the manuscript. XL, JH and LL performed the experiments and analyzed the data. All authors read and approved the final manuscript.

\section{Ethics approval and consent to participate}

The present study was approved by both Ethics Committee of Chinese PLA General Hospital and China-Japan Friendship Hospital (Beijing, China). All patients provided written informed consent for the use of their medical records and tissue specimens for research purposes. 


\section{Patient consent for publication}

Not applicable.

\section{Competing interests}

The authors declare that they have no competing interests.

\section{References}

1. Bizama C, García P, Espinoza JA, Weber H, Leal P, Nervi B and Roa JC: Targeting specific molecular pathways holds promise for advanced gallbladder cancer therapy. Cancer Treat Rev 41 222-234, 2015.

2. Randi G, Malvezzi M, Levi F, Ferlay J, Negri E, Franceschi S and La Vecchia C: Epidemiology of biliary tract cancers: An update. Ann Oncol 20: 146-155, 2009.

3. Goetze TO: Gallbladder carcinoma: Prognostic factors and therapeutic options. World J Gastroenterol 21: 12211-12217, 2015.

4. Hundal R and Shaffer EA: Gallbladder cancer: Epidemiology and outcome. Clin Epidemiol 6: 99-109, 2014.

5. Yeh YT, Dai HY and Chien CY: Amplification of MPZL1/PZR gene in hepatocellular carcinoma. Hepatobiliary Surg Nutr 3: 87-90, 2014

6. Zhao ZJ and Zhao R: Purification and cloning of PZR, a binding protein and putative physiological substrate of tyrosine phosphatase SHP-2. J Biol Chem 273: 29367-29372, 1998.

7. Taniguchi K and Karin M: IL-6 and related cytokines as the critical lynchpins between inflammation and cancer. Semin Immunol 26: 54-74, 2014.

8. Zannettino AC, Roubelakis M, Welldon KJ, Jackson DE, Simmons PJ, Bendall LJ, Henniker A, Harrison KL, Niutta S, Bradstock KF and Watt SM: Novel mesenchymal and haematopoietic cell isoforms of the SHP-2 docking receptor, PZR: Identification, molecular cloning and effects on cell migration Biochem J 370: 537-549, 2003.

9. Zhang J, Zhang F and Niu R: Functions of Shp2 in cancer. J Cell Mol Med 19: 2075-2083, 2015.

10. Labbé DP, Hardy S and Tremblay ML: Protein tyrosine phosphatases in cancer: Friends and foes! Prog Mol Biol Transl Sci 106 253-306, 2012.

11. Huang WQ, Lin Q, Zhuang X, Cai LL, Ruan RS, Lu ZX and Tzeng CM: Structure, function, and pathogenesis of SHP2 in developmental disorders and tumorigenesis. Curr Cancer Drug Targets 14: 567-588, 2014.

12. Chan G, Kalaitzidis D and Neel BG: The tyrosine phosphatase Shp2 ( PTPN11 ) in cancer. Cancer Metastasis Rev 27: 179-192, 2008

13. Grossmann KS, Rosário M, Birchmeier C and Birchmeier W The tyrosine phosphatase Shp2 in development and cancer. Adv Cancer Res 106: 53-89, 2010.

14. Roubelakis MG, Martin-Rendon E, Tsaknakis G, Stavropoulos A and Watt SM: The murine ortholog of the SHP-2 binding molecule, PZR accelerates cell migration on fibronectin and is expressed in early embryo formation. J Cell Biochem 102: 955-969, 2007.

15. Seda Eminaga and Anton M: Bennett: Noonan syndrome-associated SHP-2/Ptpn11 mutants enhance SIRP $\alpha$ and PZR tyrosyl phosphorylation and promote adhesion-mediated ERK activation. J Biol Chem 283: 15328-15338, 2008.

16. Kusano K, Thomas TN and Fujiwara K: Phosphorylation and localization of protein-zero related (PZR) in cultured endothelial cells. Endothelium 15: 127-136, 2008

17. Jia D, Jing Y, Zhang Z, Liu L, Ding J, Zhao F, Ge C, Wang Q, Chen T, Yao M, et al: Amplification of MPZL1/PZR promotes tumor cell migration through Src-mediated phosphorylation of cortactin in hepatocellular carcinoma. Cell Res 24: 204-217, 2014.

18. Yu T, Liang L, Zhao X and Yin Y: Structural and biochemical studies of the extracellular domain of Myelin protein zero-like protein 1. Biochem Biophys Res Commun 506: 883-890, 2018.

19. Livak KJ and Schmittgen TD: Analysis of relative gene expression data using real-time quantitative PCR and the 2(-Delta Delta C(T)) method. Methods 25: 402-408, 2001
20. Ma MZ, Kong X, Weng MZ, Zhang MD, Qin YY, Gong W, Zhang WJ and Quan ZW: Long non-coding RNA-LET is a positive prognostic factor and exhibits tumor-suppressive activity in gallbladder cancer. Mol Carcinog 54: 1397-1406, 2015.

21. Caplen NJ, Parrish S, Imani F, Fire A and Morgan RA: Specific inhibition of gene expression by small double-stranded RNAs in invertebrate and vertebrate systems. Proc Natl Acad Sci USA 98: 9742-9747, 2001.

22. Yoon HA, Noh MH, Kim BG, Han JS, Jang JS, Choi SR, Jeong JS and Chun JH: Clinicopathological significance of altered Notch signaling in extrahepatic cholangiocarcinoma and gallbladder carcinoma. World J Gastroenterol 17: 4023-4030, 2011.

23. Bao RF, Shu YJ, Hu YP, Wang XA, Zhang F, Liang HB, Ye YY, Li HF, Xiang SS, Weng $\mathrm{H}$, et al: miR-101 targeting ZFX suppresses tumor proliferation and metastasis by regulating the MAPK/Erk and Smad pathways in gallbladder carcinoma. Oncotarget 7: 22339-22354, 2016.

24. Zhang P, Guo Z, Wu Y, Hu R, Du J, He X, Jiao X and Zhu X: Histone deacetylase inhibitors inhibit the proliferation of gallbladder carcinoma cells by suppressing AKT/mTOR signaling. PLoS One 10: e0136193, 2015.

25. Xie F, Xu X, Xu A, Liu C, Liang F, Xue M and Bai L: Aberrant activation of Sonic hedgehog signaling in chronic cholecystitis and gallbladder carcinoma. Hum Pathol 45: 513-521, 2014.

26. Hunter T: Tyrosine phosphorylation: Thirty years and counting. Curr Opin Cell Biol 21: 140-146, 2009.

27. Hunter T: Protein kinases and phosphatases: The yin and yang of protein phosphorylation and signaling. Cell 80: 225-236, 1995.

28. Krause DS and Van Etten RA: Tyrosine kinases as targets for cancer therapy. N Engl J Med 353: 172-187, 2005.

29. Zhao S, Sedwick D and Wang Z: Genetic alterations of protein tyrosine phosphatases in human cancers. Oncogene 34: 3885-3894, 2015.

30. Gaumann AK, Kiefer F, Alfer J, Lang SA, Geissler EK and Breier G: Receptor tyrosine kinase inhibitors: Are they Real Tumor Killers? Int J Cancer 138: 540-554, 2016.

31. Tonks NK: Protein tyrosine phosphatases-from housekeeping enzymes to master regulators of signal transduction. FEBS J 280: 346-378, 2013.

32. Julien SG, Dubé N, Hardy S and Tremblay ML: Inside the human cancer tyrosine phosphatome. Nat Rev Cancer 11: 35-49, 2011.

33. He RJ, Yu ZH, Zhang RY and Zhang ZY: Protein tyrosine phosphatases as potential therapeutic targets. Acta Pharmacol Sin 35: 1227-1246, 2014

34. Li M, Zhang Z, Li X, Ye J, Wu X, Tan Z, Liu C, Shen B, Wang XA $\mathrm{Wu} \mathrm{W}$, et al: Whole-exome and targeted gene sequencing of gallbladder carcinoma identifies recurrent mutations in the ErbB pathway. Nat Genet 46: 872-876, 2014.

35. Zhao R, Fu X, Teng L, Li Q and Zhao ZJ: Blocking the function of tyrosine phosphatase SHP-2 by targeting its Src homology 2 domains. J Biol Chem 278: 42893-42898, 2003.

36. Eminaga S and Bennett AM: Noonan syndrome-associated SHP-2/Ptpn11 mutants enhance SIRPalpha and PZR tyrosyl phosphorylation and promote adhesion-mediated ERK activation. J Biol Chem 283: 15328-15338, 2008.

37. Chen L, Wang ZW, Zhu JW and Zhan X: Roles of cortactin, an actin polymerization mediator, in cell endocytosis. Acta Biochim Biophys Sin (Shanghai) 38: 95-103, 2006.

38. Sung BH, Zhu X, Kaverina I and Weaver AM: Cortactin controls cell motility and lamellipodial dynamics by regulating ECM secretion. Curr Biol 21: 1460-1469, 2011.

39. Eckert MA, Lwin TM, Chang AT, Kim J, Danis E, Ohno-Machado L and Yang J: Twist1-induced invadopodia formation promotes tumor metastasis. Cancer Cell 19: 372-386, 2011.

40. Zhao R, Guerrah A, Tang H and Zhao ZJ: Cell surface glycoprotein PZR is a major mediator of concanavalin A-induced cell signaling. J Biol Chem 277: 7882-7888, 2002.

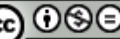

This work is licensed under a Creative Commons Attribution-NonCommercial-NoDerivatives 4.0 International (CC BY-NC-ND 4.0) License. 